\title{
Introductory physics students' insights for improving physics culture
}

\author{
Acacia Arielle Evans ${ }^{1}$, Kai S. Bretl ${ }^{1}$, Amad Ross $^{2}$, and Abigail R. Daane ${ }^{1}$ \\ ${ }^{l}$ Department of Physics, South Seattle College, $600016^{\text {th }}$ Ave. SW, Seattle, WA 98106, USA \\ ${ }^{2}$ Columbia College, Columbia University, 1130 Amsterdam Avenue, New York, NY 10027, USA
}

Ethnic and Racial Minorities (ERM) and women are underrepresented in classrooms and the field of physics. We can work to address this disparity by empowering students to change the physics culture within their own spheres of influence. Students in introductory, calculus-based physics classes from both two and four-year institutions participated in lessons from the Underrepresentation Curriculum, a freely available curriculum designed to bring social justice conversations to the classroom. Post unit, students brainstormed ideas about how to raise awareness of, and ultimately remove this inequity. We coded students' responses grouping analogous key words and phrases. Our analysis showed that students from both institutions generated similar sets of propositions. Their responses included having intentional conversations about equity issues and actively learning about their own biases. By following students' suggestions, we can create a more inclusive and diverse physics community. 


\section{INTRODUCTION}

The field of physics has a big problem: physics has a disproportionately low number of Ethnic \& Racial Minorities (ERM) [1] and women [2] and it does not seem to be improving. The structures in physics culture (e.g., cultural practices for college admissions, hiring processes, grant applications, teaching practices, instructor bias, larger societal influences, etc.) continue to deter members of historically excluded groups with great success. We believe that there are both consequential and deontological justifications for changing this underrepresentation and deterrence of certain groups. First, the consequences for this lack of representation mean that we are restricting the formation of hypotheses, minimizing our foci of research and curricular topics, and reducing our opportunities to solve the current world challenges that need talented, deep-thinking physicists [3]. In short, physics is stymied because of the lack of minds and resources it supports currently. Second, we believe that the deontological justification is simply that it is wrong to condone and allow power structures and cultural practices that marginalize some and empower others.

By giving students the tools to become aware of and combat this disproportionality, we can work together to make physics a field of equity and inclusivity. A first step to changing who constitutes physics is changing physics culture. As instructors and research mentors, we can begin to do this from many angles. In this paper, we present one pathway toward improving the physics culture - learning from the insights of students.

\section{CONTEXT \& METHODS}

Students in introductory calculus-based physics classes from both two and four-year institutions in the Pacific NW participated in the Underrepresentation Curriculum (URC) [4], a freely available, modular, student-centered curriculum designed to examine and address equity and inclusion in science. The URC offers students the opportunity to engage explicitly in conversations about who does physics and what structures are in place that keep the physics population different from the general population. The curriculum has three major goals:

1) Students compare graphs that highlight the underrepresentation of marginalized groups in the physics community.

2) Students acknowledge that physics has subjective components, resulting in many systemic reasons that the disparity in numbers exists.

3) Students brainstorm ways in which the culture of physics can be ruptured to create a new space for equity.

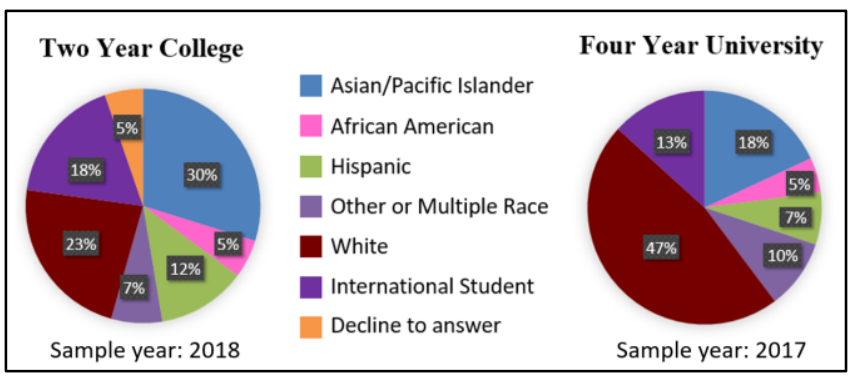

FIG 1. Racial demographics of sample physics classes at both institutions.

Students in this convenience sample participated in these lessons in the middle of a yearlong introductory sequence taught in small (20-60 students per class) interactive classroom settings in 2017 (4-yr) \& 2018-2019 (2-yr). These implementations of the URC were for $~ 220-300$ instructional minutes by white, female instructors described elsewhere [5], with many implementations possible depending on context (e.g., [6]). Figure 1 shows a sample year of the racial demographics of the students taught at each institution.

In both settings, we found students had many "resources" (i.e., productive ideas for learning about equity) [7] that have significant meaning for our community. Previous research highlights students' views on structural bias, inequity, and the disproportionate representation of certain racial groups in physics during the URC $[8,9]$. Here, we present students' ideas, gathered from small group responses to the final URC discussion question, "What can we do now?" Students brainstormed several ideas about what actions they and others can take to raise awareness of, and ultimately remove, this inequity problem. We used an emergent coding process for students' responses, grouping analogous key words and phrases [10]. For example, the quotes "Don't be afraid to talk about [the problem]" (4-yr) and "Explicit and careful communication - not mindless communication" (2-yr) both contain phrases relating to discourse so they were categorized in the "Create Conversations" theme. We individually coded each response then met iteratively to create agreed-upon categories. Between three coders, our interrater reliability rating was $94 \%$ and we settled all disagreements through discussion. We coded 200 responses into the categories out of 211 total responses. Of the remaining responses, three were not action-items and eight were too general to categorize.

We looked for statistically significant differences between the numbers of responses in each theme from both institutions using Fisher's Exact Test. We recognize that this information is limited because the test does not report effect size. However, this test gives a general picture of the differences between responses based on our emergent categories. Another limitation is that we interpreted these responses at face value and students may have meant 


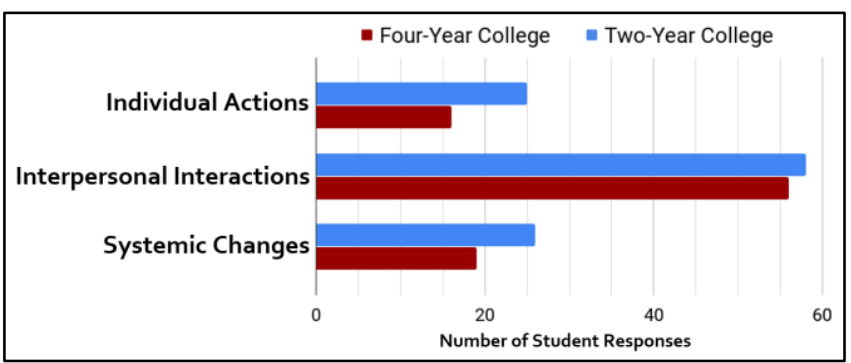

FIG 2. Total number of coded student generated responses in each emergent theme by institution (no statistical difference).

something different in the context of their small group conversations. We do not make the claim that the use of the URC influenced the creation of students' responses and we acknowledge that the generation of many of these brilliant ideas may have been possible without the lessons.

\section{RESULTS}

The total number of students' responses from both institutions were organized into three main themes: Individual Actions, Interpersonal Interactions, and Systemic Changes (Fig. 2). Within each larger theme, we identified two subthemes (Table I); all subthemes were found in both institutions. Our analysis showed that the percentage of students' responses in each subtheme were not statistically different across both institutions, with the exception of the Develop Coping Strategies theme (Fig. 3).

\section{A. Individual Actions}

A typical response to anti-racist education is to stay within the boundaries of internalized dominance, focusing on one's own lack of ability to make changes [11]. We found that students' responses contrasted this helplessness and lack of ownership of individual contributions: many responses focused on the individual's responsibility to increase understanding of how their actions affect themselves and those around them. Students in these courses identified "becoming aware" of equity issues in physics and "developing coping strategies" as ways to start improving the culture in and out of the classroom.

TABLE I. Themes of student-generated responses.

\begin{tabular}{ll}
\hline \hline Main Themes & Subthemes \\
\hline A. Individual & 1. Become Aware \\
Actions & 2. Develop Coping Strategies \\
B. Interpersonal & 1. Value People \\
Interactions & 2. Create Conversations \\
C. Systemic & 1. Lift Up ERM/Women \\
Changes & 2. Change Systems \\
\hline \hline
\end{tabular}

\section{Become Aware}

Many student groups explicitly stated actions about increasing self-awareness and taking initiative to learn more about their own personal bias. We agree that the physics community needs to critically examine the ways in which we construct and uphold systemic power structures [12]. Students from both the two-year (2-yr) and four-year (4-yr) institutions wrote responses such as:

"A good first step is acknowledging where implicit bias exists and being conscious of it. Like any problem, realizing that there IS a problem is an important first step" (2-yr),

"Recognize the intent and reasons why we do things" (4-yr), and

"Keep yourself responsible" (4-yr).

These three quotes highlight the emphasis on introspection in this category. By identifying and reflecting on their own inevitable personal bias, students can better understand how their actions affect those in the community from both systemically dominant and nondominant groups. Taking this a step further and changing personal actions to address personal bias will make a positive change in the immediate atmosphere of the classrooms and larger community.

Other student groups, particularly those at the four-year institution, emphasized learning more about equity and bias beyond the URC lessons:

"Learn about stereotypes" (4-yr) and

"Educate yourself [about these issues]" (4-yr).

The responses focused on actions to improve the physics culture, and thus the latter quote fits in this category because it answers the specific question in this context. A difference in responses between the two-year and four-year institutions was that the two-year responses did not mention additional learning about stereotypes beyond the URC experience. The two-year college students may have been more familiar with the concept of stereotype threat because of their background

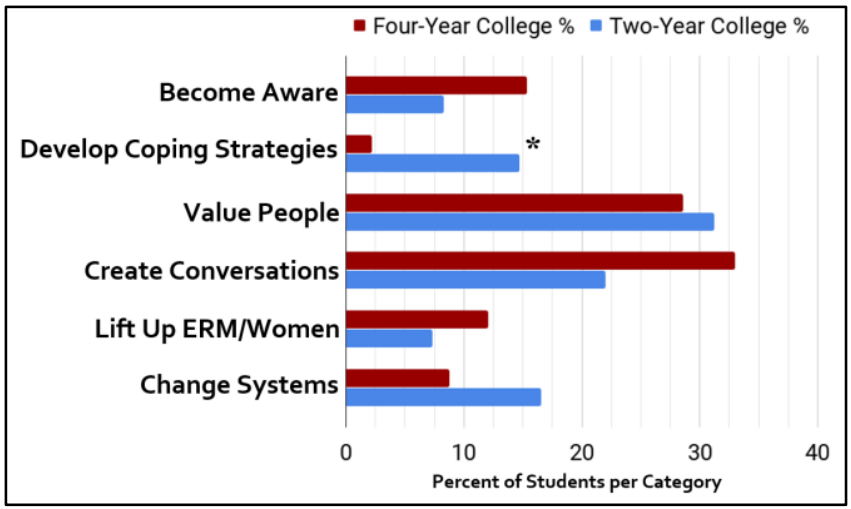

FIG 3. Percentage of emergent student generated solutions by institution per category, $* p<0.05$, Fisher's exact test. 
and context. Data was collected at the 4-yr institution in 2017 , then one and two years later at the 2 -yr college. The change in socio-political climate during the 1-2 years between implementations may have increased awareness of structural barriers in education for all students. Regardless, both populations emphasized that knowledge of personal bias, larger structural bias, and equity issues in physics may increase individuals awareness of why the culture needs disrupting - a first step towards change.

\section{Developing Coping Strategies}

Student ideas in the "develop coping strategies" category discussed how to respond to stereotype threat, microaggressions, imposter phenomenon, bias, etc. in selfsustaining ways. Developing coping strategies was the only category with a statistical significance between institutions, ( $p<0.05$, Fisher's exact test). Two-year colleges generated significantly more developing coping strategy responses than the four-year university. Two-year college students wrote,

"Be forgiving to people who say things either from ignorance or to directly offend you,"

"Find ways to remain resilient and maintain selfconfidence," and

"Have study groups of women/minorities."

Four-year university students wrote,

"Respond to hate with love," and

"Create study groups."

The quotes shared here are a small portion of what students wrote in the two-year college, while the quotes from the fouryear university were the only two quotes that fit into the Develop Coping Strategies category. This category represents an important distinction between the actions that emerged at the different institutions. It may be that the students for whom this category is relevant did not feel as comfortable sharing their actions about coping in the predominantly white (4-yr) institution. Regardless, the difference between responses highlights the importance of attending to your student population when applying curricula [13]. The student's experience and context influences the relevance and outcome of lessons, especially with the URC.

\section{B. Interpersonal Interactions}

Responses in this category were the most common among student groups in both institutions. Students described ways to connect positively with people by conversing with and respecting each other in every aspect of life.

\section{Value People}

One category that appeared across many groups was to value people. We included a wide range of responses in this category that connected through respect and genuine care for others - especially those who have different identities, ideas, experiences, or backgrounds. Students stated,

"Create positive connections whenever possible" (2-yr),

"Take care of each other" (2-yr),

"Help classmates who may be struggling” (4-yr), and

"Step outside of your social circle" (4-yr).

These ideas illustrate that it takes direct action to bring positivity to one's surroundings. Simply being is not enough to cause change. Students wrote ideas about embracing, respecting, and including people from other cultures and backgrounds. They included steps towards creating a sense of belonging for others, feeling validated by being kind, keeping your mind open to new ideas, and seeking out new experiences.

\section{Create Conversations}

Some student ideas aligned with research showing that by simply having conversations about the disparity in physics across certain groups, the likelihood increases that members of nondominant groups will continue [14]. This category encompasses myriad recommendations that focused on the importance of continuing the discourse - in the classroom and beyond. Students wrote:

"If you hear someone say something offensive, don't be silent” (2-yr),

"Maintain ... open discussion of biases to continue the improvement of equality and ensure no turning back into ignorance of biases" (2-yr) and

"Reach out to kids at a young age" (4-yr).

These quotes show different perspectives/actions that can positively affect the environment of physics using different communication approaches. Several other responses include increased dialogue about issues of equity in physics by applying strategies of listening and speaking up when the conversation gets tough. Students also wrote about taking initiative when bringing awareness to people outside the classroom.

\section{Systemic Changes}

Systemic changes are larger changes that affect a greater number of people per change. Student responses under Systemic Changes showed different ways to create structural changes inside and outside of a school setting, including a focus directly on improving the experiences and opportunities for ERM and women. 


\section{Lift Up ERM/Women}

Student ideas in this category focused on supporting systemically nondominant groups in various ways. Students wrote,

"Become the top-level (top of the field) person and work to support the people in need" (2-yr),

"Promote minority role models" (4-yr), and

"Include forms of representation on posters, playing cards, videos, etc." (2-yr).

These three quotes show the importance of having visible examples of role models for students from systemically nondominant groups. Having examples of people in the field who come from similar backgrounds can increase sense of belonging, empower individuals and decrease feelings of isolation. Note that the mention of "playing cards" came from a particular classroom, where the instructor uses playing cards as a tool for random group assignment. The playing cards highlighted physicists from systemically nondominant backgrounds. Student groups also had ideas such as,

"Support local STEAM clubs; inspire all children" (2-yr), and

"Mentor programs for minorities by minorities" (4-yr). These quotes focus on supporting ERM and women through supportive programs. The "inspire all children" aspect of the second quote is inferred to focus on ERM and women, based off the context of the question students were asked to respond to from the URC.

\section{Change Systems}

Although the idea of changing systems was not commonly generated in either institution, some groups mentioned changing systems in and out of schools. A change in the system could be,

"[Re]phrasing of tests to not give one group of people an advantage” (2-yr).

An example of this would be way the SAT and ACT tests are written and organized. According to Steele, these tests are not controlled tests that quantify intelligence - they just measure how well one can take a test. "Standardized tests can not accurately measure intellectual merit because racial and gender stereotypes interfere with the intellectual functioning of those taking the tests" [15]. Another idea from a student is to have,

"Sensitivity training for faculty" (4-yr).

From the perspectives of authors 1-3, all current students (two from systemically nondominant groups), simply having a professor who is aware of sensitivities and their own biases can work wonders for students' class experiences.

\section{DISCUSSION \& CONCLUSIONS}

Our analysis highlights that students generate productive ideas about how to approach the problem of an exclusive and inequitable physics culture. In both institutional contexts, which varied greatly in their racial demographics, we found student responses to be generally positive. The first category - become aware of internal biases and continuously working to counteract them - can be a particularly important first step. Looking inward can create motivation and awareness that supports both a change in our interactions with others and the larger decisions we make towards changing the physics culture. We suspect that this category was largely present because of the just-prior implementation of the URC and the current inequities in physics.

We encourage students, instructors, and administrators in school systems and the larger physics community alike to work together to change structures that maintain exclusivity and inequity. This can begin by providing students the opportunity to process and share their ideas. We share these results from students, not just to show that students have ideas for action, but because physics researchers and instructors can use these recommendations from students in their research practices and classes. Second, instructors and researcher-mentors can create spaces to listen to their own students, who may have other needs in their context to make physics more inclusive. For example, we found that in our two-year college setting, significantly more mentions of the need to develop coping mechanisms arose. Third, instructors can support their colleagues and students in becoming agents of change through increased discussions of these issues.

After this analysis, we realized that this implementation of the URC could do a better job of supporting students in reflecting on how to contribute to systemic changes in physics. This could include providing specific steps for creating or joining inclusive clubs at school, encouraging student involvement in hiring or elective processes in schools, and increasing awareness and action on current societal issues and political legislation that relates to education and scientific research. Additionally, depending on the context, the student population will need different areas of support following the URC unit. Our hope is that by compiling and sharing student responses, current and future members of the physics community can use these ideas to create a more inclusive physics culture.

\section{ACKNOWLEDGEMENTS}

The authors wish to thank the student participants at both institutions and Sofia Herrera, Dr. Elizabeth Schoene, Quynh Dang, and Jake Ashcraft for their support on this project. This material is based upon work supported by the National Science Foundation under grant no. S-STEM1643580. 
[1] AIP Statistics. "The Proportion of Physics Bachelor's Degrees Awarded to African-Americans and HispanicAmericans." American Institute of Physics, 10 Apr. 2019, www.aip.org/statistics/data-graphics/proportionphysics-bachelors-degrees-awarded-african-americansand-hispanic.

[2] AIP Statistics. "Percent of Bachelor's Degrees Earned by Women in Selected Fields, Classes of 1981 through 2016." American Institute of Physics, 22 Apr. 2019, www.aip.org/statistics/data-graphics/percentbachelors-degrees-earned-women-selected-fieldsclasses-1981-through.

[3] J. Nespor, Knowledge in motion, London: Falmer Press (1994).

[4] http://www.underrep.com. Retrieved 6/10/2019.

[5] A.R. Daane, S.R. Decker, and V. Sawtelle, Teaching About Racial Equity in Introductory Physics Courses, Phys. Teach. 55, 328 (2017). 10.1119/1.4999724

[6] M. Rifkin, "Addressing underrepresentation: Physics teaching for all," Phys. Teach. 54, 72 (Feb. 2016).

[7] D. Hammer, "Resources paper Student resources for learning introductory physics," Phys. Educ. Res., Am. J. Phys. Suppl. 68 7, (2000).
[8] S.R. Decker and A.R. Daane, "Teaching about inequity: Shifts in student views about diversity in physics," in 2017 PERC (American Association of Physics Teachers, 2017), pp. 108-111.

[9] A.R. Daane and V. Sawtelle, "Student discourse about equity in an introductory college physics course," in 2016 PERC (American Association of Physics Teachers, 2016), pp. 88-91.

[10] K. Krippendorff, Content Analysis: An Introduction to Its Methodology (Sage, CA, 2013), 3rd edn.

[11] R. DiAngelo, "White Fragility," International Journal of Critical Pedagogy, 3 (3) (2011) pp 54-70.

[12] B. Applebaum, "Critical Whiteness Studies," Oxford Res. Encycl. of Educ., (2016).

[13] S. Kanim, \& X.C. Cid, "The demographics of physics education research," Arxiv preprint (2017). https://arxiv.org/ftp/arxiv/papers/1710/1710.02598.pdf.

[14] R. M. Lock and Z. Hazari, "Discussing underrepresentation as a means to facilitating female students' physics identity development," Phys. Rev. PER 12, 020101 (2016).

[15] Stanford University News Service. Stereotypes Found to Affect Performance on Standardized Test, (1996). 